\title{
Front Matter: Volume 7651
}

, "Front Matter: Volume 7651," Proc. SPIE 7651, International Conference on Space Information Technology 2009, 765101 (2 April 2010); doi: $10.1117 / 12.862352$

SPIE Event: International Conference on Space Information Technology 2009, SPIE. 2009, Beijing, China 


\title{
PROCEEDINGS OF SPIE
}

\section{International Conference on Space Information Technology 2009}

\author{
Xingrui Ma \\ Baohua Yang \\ Ming Li \\ Editors
}

26-27 November 2009

Beijing, China

Organized by

Space Star Technology Company, Ltd. (China)

Sponsored by

China Academy of Space Technology (China)

Cosponsored by

The Second Academy of China Aerospace Science and Industry Corporation (China)

Huazhong University of Science and Technology (China)

Published by

SPIE

Volume 7651

Proceedings of SPIE, 0277-786X, v. 7651 
The papers included in this volume were part of the technical conference cited on the cover and title page. Papers were selected and subject to review by the editors and conference program committee. Some conference presentations may not be available for publication. The papers published in these proceedings reflect the work and thoughts of the authors and are published herein as submitted. The publisher is not responsible for the validity of the information or for any outcomes resulting from reliance thereon.

Please use the following format to cite material from this book:

Author(s), "Title of Paper," in International Conference on Space Information Technology 2009, edited by Xingrui Ma, Baohua Yang, Ming Li, Proceedings of SPIE Vol. 7651 (SPIE, Bellingham, WA, 2010) Article CID Number.

ISSN 0277-786X

ISBN 9780819480798

Published by

SPIE

P.O. Box 10, Bellingham, Washington 98227-0010 USA

Telephone +1 3606763290 (Pacific Time) · Fax +1 3606471445

SPIE.org

Copyright (@ 2010, Society of Photo-Optical Instrumentation Engineers

Copying of material in this book for internal or personal use, or for the internal or personal use of specific clients, beyond the fair use provisions granted by the U.S. Copyright Law is authorized by SPIE subject to payment of copying fees. The Transactional Reporting Service base fee for this volume is $\$ 18.00$ per article (or portion thereof), which should be paid directly to the Copyright Clearance Center (CCC), 222 Rosewood Drive, Danvers, MA 01923. Payment may also be made electronically through CCC Online at copyright.com. Other copying for republication, resale, advertising or promotion, or any form of systematic or multiple reproduction of any material in this book is prohibited except with permission in writing from the publisher. The CCC fee code is 0277-786X/10/\$18.00.

Printed in the United States of America.

Publication of record for individual papers is online in the SPIE Digital Library.

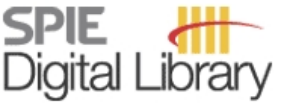

SPIEDigitalLibrary.org

Paper Numbering: Proceedings of SPIE follow an e-First publication model, with papers published first online and then in print and on CD-ROM. Papers are published as they are submitted and meet publication criteria. A unique, consistent, permanent citation identifier (CID) number is assigned to each article at the time of the first publication. Utilization of CIDs allows articles to be fully citable as soon they are published online, and connects the same identifier to all online, print, and electronic versions of the publication. SPIE uses a six-digit CID article numbering system in which:

- The first four digits correspond to the SPIE volume number.

- The last two digits indicate publication order within the volume using a Base 36 numbering system employing both numerals and letters. These two-number sets start with 00, 01, 02, 03, 04 , 05, 06, 07, 08, 09, OA, OB ... 0Z, followed by 10-1Z, 20-2Z, etc.

The CID number appears on each page of the manuscript. The complete citation is used on the first page, and an abbreviated version on subsequent pages. Numbers in the index correspond to the last two digits of the six-digit CID number. 


\section{Contents}

\section{Part One}

xv Conference Committee

xix Introduction

DEEP SPACE EXPLORATION AND COMMUNICATION TECHNOLOGY/ACQUISITION, PROCESSING, AND APPLICATION TECHNOLOGY OF SPACE INFORMATION

765102 ML-IKE: a multi-layer IKE protocol for TCP performance enhancement in wireless networks [7651-60]

Y.-H. Zhang, B.-W. Cheng, Space Star Technology Co., Ltd. (China) and Peking Univ. (China); S.-H. Qing, Peking Univ. (China); G.-N. Zou, Space Star Technology Co., Ltd. (China); W.-P. Wen, Peking Univ. (China)

765103 A WebGIS system on the base of spatial data processing system for land application [7651-13]

D. WU, Q. Wang, H. Wang, Z. Wang, Southeast Univ. (China)

765104 A 2D vector map watermarking algorithm resistant to simplication attack [7651-66] C. Wang, Wuhan Univ. (China) and Shihezi Univ. (China); B. Liang, Q. Zhao, Z. Qiu, Shihezi Univ. (China); Y. Peng, L. Yu, Wuhan Univ. (China)

765105 Australian fire monitoring and assessment using CCD and IRS data of HJ-1-A/B [7651-166] S. Liu, S. Yang, National Disaster Reduction Ctr. of China (China)

765106 Research on imaging algorithm of target detection based on BD signals [7651-167] J. Yang, W. Liang, J. Wang, L. YU, National Key Lab. of Science and Technology on C4ISR (China)

765107 Study on the spectrum reconstruction and relative radiometric calibration of interference imaging spectrometer [7651-122]

L. Yao, W. Zhao, S. Fan, Space Star Technology Co., Ltd. (China)

765108 The scheme of LLSST based on inter-satellite link for planet gravity field measurement in deep-space mission [7651-68]

Y. Yang, X. Li, L. Liu, Univ. of Electronic Science and Technology of China (China)

765109 Satellite image compression based on fractal theory [7651-95]

Y. Zhou, P. Liu, Y. Huang, China Academy of Space Technology (China)

7651 OA The method of low-orbit satellite ambiguity fixing on network RTK [7651-111]

S. Pan, Q. Wang, S. Wang, F. Ke, Southeast Univ. (China)

7651 OB Monte Carlo simulation method of atmosphere-ocean laser transmission [7651-48]

Y. Dong, C. Li, R. Huang, Huazhong Univ. of Science and Technology (China) 
7651 OC Simulation of space-based optical surveillance visible image [7651-53] H. Sun, Y. Han, Y. Li, Academy of Equipment Command \& Technology (China)

7651 OD Research on space object active detection ladar system imaging simulation [7651-58] H. Sun, Y. Han, Academy of Equipment Command \& Technology (China)

7651 OE Tropospheric zenith delay monitoring in GPS precise point positioning [7651-34] Q. Zhu, Z. Wu, Xidian Univ. (China); Z. Zhao, China Research Institute of Radio Wave Propagation (China)

7651 OF Development tendency and key technology of deep space exploration's sample return capsule [7651-171]

Z. Xing, H. Zhao, B. Wen, Beijing Institute of Spacecraft System Engineering (China)

7651 OG A method for classification training samples spatial-time expanding of remote sensing image [7651-07]

G. Ren, Ocean Univ. of China (China) and First Institute of Oceanography (China); J. Zhang, Y. Ma, First Institute of Oceanography (China) and Key Lab. of Marine Science and Numerical Modeling (China); R. Zheng, Ocean Univ. of China (China)

$7651 \mathrm{OH}$ An automatic registration method for different temporal remote sensing images based on improved Fourier-Mellin algorithm [7651-03]

R. Li, Henan Univ. (China) and Institute of Geographical Sciences and Natural Resources Research (China); J. Sun, F. Yin, Institute of Geographical Sciences and Natural Resources Research (China); F. Guo, Institute of Automation (China); W. Wang, Henan Univ. (China) and Institute of Geographical Sciences and Natural Resources Research (China)

7651 ol An image fusion method based on wavelet transform and ICM [7651-92] H. Wang, M. Li, Y. Li, K. Zhang, Northwestern Polytechnical Univ. (China)

7651 0J A modified cadastral survey system based on GPS/PDA [7651-110] H. Wang, Q. Wang, X. Wu, Southeast Univ. (China)

7651 OK The study on the synthesis approximate algorithm of GPS height conversion [7651-33] X. Wu, Q. Wang, B. Gao, H. Liang, Southeast Univ. (China)

7651 OL Design of the automatic landing inversion flight control system based on neural network compensation for UAV [7651-91]

Y. Chen, W. Yang, Northwestern Polytechnical Univ. (China)

7651 OM Image fusion algorithm based on energy of Laplacian and PCNN [7651-132]

M. Li, Northwestern Polytechnical Univ. (China) and Xi'an Shiyou Univ. (China); H. Wang, Y. Li, K. Zhang, Northwestern Polytechnical Univ. (China)

7651 ON Automatic target tracking in infrared image sequences using ensemble distance metric [7651-59]

Z. Wang, G. Yang, North China Electric Power Univ. (China)

765100 A method of phase estimation for sinusoid signal based on correlation algorithm [7651-38] X. Chang, X. Zhang, Q. Zhang, Beijing Univ. of Aeronautics and Astronautics (China) 
$7651 \mathrm{OP} \quad$ A study on two-level network monitoring system for space exploration [7651-121]

Y. Ding, K. Hu, L. Chen, Beihang Univ. (China)

$76510 Q \quad$ Bit assignment optimization for ROI of CCSDS image compression standards based on DWT [7651-73]

J. Zhang, H. Zhao, J. Ma, Y. Cui, Harbin Institute of Technology (China)

7651 OR Development of assistant and decision-making system of site selection for supermarket based on MapX [7651-123]

S.-D. Wang, J.-H. Cheng, X. Ma, Henan Polytechnic Univ. (China)

7651 OS An improved FFT acquisition method for weak signal in deep-space communications [7651-54]

X. Pan, Beijing Institute of Technology (China); M. Chen, Beijing Research Institute of Telemetry (China)

7651 OT A method of content-based image analysis using SVM classifier in data catalogue and archive system of remote sensing satellite [7651-102]

X. Wang, S. Fan, Space Star Technology Co., Ltd. (China)

7651 OU SAR image segmentation based on the two-dimensional Pseudo Wigner-Ville Distribution [7651-56]

C. Liu, Y. Li, K. Zhang, Northwestern Polytechnical Univ. (China)

7651 OV Satellite antenna layout and optimization in electromagnetic compatibility design [7651-142]

J. Zhang, S. Xie, Y. Liu, Beijing Univ. of Aeronautics and Astronautics (China)

7651 OW Search for random fluctuations in periods of Cepheids [7651-148]

L. N. Berdnikov, Sternberg Astronomical Institute (Russian Federation); I. R. Stevens, The Univ. of Birmingham (United Kingdom)

$76510 X \quad H o w$ human resource organization can enhance space information acquisition and processing: the experience of the VENESAT-1 ground segment [7651-126]

R. Acevedo, The Bolivarian Agency for Space Activities (Venezuela); N. Orihuela, Central Univ. of Venezuela (Venezuela); R. Blanco, The Bolivarian Agency for Space Activities (Venezuela); F. Varela, Beihang Univ. (China) and Central Univ. of Venezuela (Venezuela); E. Camacho, M. Urbina, L. G. Aponte, L. Vallenilla, L. Acuña, R. Becerra, T. Tabare,

E. Recaredo, The Bolivarian Agency for Space Activities (Venezuela)

7651 OY Application of rough set theory in geodesic data processing [7651-109]

S. Gu, Huazhong Univ. of Science and Technology (China) and Beijing Aerospace Control Ctr. (China); J. Wei, Huazhong Univ. of Science and Technology (China); J. Guo, Beijing Aerospace Control Ctr. (China)

$76510 Z$ Effective spatial database support for acquiring spatial information from remote sensing images [7651-31]

P. Jin, S. Wan, L. Yue, Univ. of Science and Technology of China (China) 
765110 Discussion on spatio-temporal data model of mining-land use change [7651-84] C. Liu, Henan Polytechnic Univ. (China); H. Zhang, Luoyang Institute of Science and Technology (China); S. Wang, Henan Polytechnic Univ. (China)

\section{SATELLITE COMMUNICATION TECHNOLOGY/SATELLITE APPLICATION TECHNOLOGY}

765111 Design and implementation of 800Mbps 16-QAM pulse shaping filter in high speed satellite transmission [7651-23]

J. Hu, Ctr. for Space Science and Applied Research (China) and Graduate Univ. of the Chinese Academy of Sciences (China); X. Cheng, W. Xiong, X. Chen, Ctr. for Space Science and Applied Research (China)

765112 The development and inspiration of space-based information system [7651-168] W. Liang, Z. Duan Mu, J. Gao, National Key Lab. of Science and Technology on C4ISR (China)

765113 Study on outgoing longwave radiation variations associated with strong earthquake [7651-144]

F. Jing, Institute of Remote Sensing Applications (China), Graduate Univ. of the Chinese Academy of Sciences (China), and Institute of Earthquake Science (China); X. Gu, Institute of Remote Sensing Applications (China); X. Shen, Institute of Earthquake Science (China)

765114 An air traffic flow management method based on mixed genetic algorithms [7651-174] Y. Fu, Air Force Equipment Academy (China)

765115 A Ku band circularly polarized U-slot antenna [7651-146] L. Han, N. Liu, Space Star Technology Co., Ltd. (China)

765116 Analysis of effect of electromagnetic interference on detection data from the data transmitting system of the electromagnetic satellite [7651-24] R. Yan, Institute of Engineering Mechanics (China) and Institute of Crustal Dynamics (China); J.-F. Zhang, Institute of Crustal Dynamics (China); Y. An, P.-L. Wang, Y.-R. Liu, Academy of Opto-Electronics (China)

765117 Mitigating congestion in wireless ad hoc networks by using a potential-based routing algorithm [7651-16]

W. Wu, Z. Zhang, D. Qin, C. He, Harbin Institute of Technology (China)

765118 Performance of $\mathbf{M}$-ary spread spectrum communication system present in multipath [7651-120]

B. Wang, Space Star Technology Co., Ltd. (China); L. Zhang, Univ. of Science and Technology Beijing (China)

765119 Research on synthetic decision support technology based on data farming [7651-30] H. Shi, Z. Yu, W. Li, Northwestern Polytechnical Univ. (China)

7651 1A Dynamic network management and service integration for airborne network [7651-76] W. Pan, W. Li, Northwestern Polytechnical Univ. (China) 
7651 1B An adaptive detection model of moving dim targets based on energy difference between frames [7651-114]

Y. Liu, Nanchang Institute of Technology (China); G. Yan, Huazhong Univ. of Science and Technology (China)

7651 1C Research of STBC in free space optical communications system [7651-41]

T. Deng, K. Peng, Y. Lu, Huazhong Univ. of Science and Technology (China)

7651 1D High efficiency and dual polarized array antenna for satellite communication application [7651-152]

N. Liu, Space Star Technology Co., Ltd. (China)

$76511 \mathrm{E} \quad$ Numerical simulation of thermal effects on a $2 \mathrm{~m}$ space telescope [7651-09]

Y. Gong, F. Zhao, L. Zhang, H. Xiang, Beijing Technology and Business Univ. (China); P. Wang, Beijing Institute of Technology (China)

7651 iF Unscented Kalman filter with open-loop compensation for high dynamic GNSS carrier tracking [7651-19]

W. Wang, X. Chen, S. Han, W. Meng, Harbin Institute of Technology (China); Y. Zhang, Space Star Technology Co., Ltd. (China)

7651 1G An FPGA-based DS-CDMA multiuser demodulator employing adaptive multistage parallel interference cancellation [7651-15]

X. Li, Z. Song, Y. Zhan, Q. Wu, Space Star Technology Co., Ltd. (China) and Beijing Institute of Technology (China)

7651 1H An efficient decoding for low density parity check codes [7651-02]

L. Zhao, X. Zhang, M. Zhu, Beijing Univ. of Aeronautics and Astronautics (China)

765111 Interoperation between AODV protocol and AOHR protocol for mobile ad hoc networks [7651-125]

S. Wu, C. Wang, J. Zhang, Harbin Institute of Technology (China)

7651 1 J Max-min energy-aware hybrid routing protocol for ad hoc networks [7651-124]

S. Wu, C. Wang, X. Bai, Harbin Institute of Technology (China)

7651 1K Enhanced reliable transmission control protocol for spatial information networks [7651-74]

Z. Qin, J. Zhang, J. Wang, Sichuan Univ. (China)

$76511 \mathrm{~L}$ Intrusion tolerant model for spatial information networks based on defense-in-depth [7651-67]

J. Zhang, Z. Qin, J. Wang, Sichuan Univ. (China)

$76511 \mathrm{M}$ Comparison of two MAC protocols based on LEO satellite networks [7651-170]

M. Guan, R. Wang, Shenzhen Institute of Information Technology (China)

$76511 \mathrm{~N}$ The atmospheric conditions of the Maidanak Observatory in Uzbekistan for ground-based observations [7651-137]

S. Ilyasov, Y. Tillayev, Ulugh Beg Astronomical Institute (Uzbekistan) 
765110 Space based remote sensing applications for archaeology [7651-139]

M. B. Rajani, Indian Institute of Science, Bangalore (India)

7651 1P A pricing scheme to maximize social welfare in communication networks [7651-130] J. Estévez, H. G. Xiong, Q. Gao, E. Guerrero, Beijing Univ. of Aeronautics and Astronautics (China)

$76511 Q \quad$ Research on carrier noise ratio calculation method in satellite communication link [7651-163]

M. Zhu, C. Zhang, S. Min, Space Star Technology Co., Ltd. (China)

7651 1R A novel feature link quality analysis method applied in satellite adaptive frequency hopping communication [7651-89]

D. Wu, B. Wang, Z. Han, C. Xiao, Space Star Technology Co., Ltd. (China)

7651 is Method to calculate bandwidth of VLF transmitting antenna based on MSK power spectrum [7651-21]

Y.-H. Dong, G.-Q. Jin, Naval Univ. of Engineering (China)

7651 1T Precise relative positioning of formation of spacecraft using GPS [7651-177]

Z. Liang, T. Ju, Space Star Technology Co., Ltd. (China)

$76511 \mathrm{U} \quad$ Remote sensing satellite data integration and service technology [7651-96]

Y. Lee, F. Wang, X. Lee, Z. Shi, X. Zhou, Space Star Technology Co., Ltd. (China)

7651 IV Study on resource management algorithm based on infinitesimal perturbation analysis for communication networks [7651-140]

Y. Song, Jilin Normal Univ. (China); Z. Zhang, Changchun Teachers College (China); N. He, Institute of Equipment and Command Technology (China) and People's Liberation Army (China)

7651 IW A novel circularly polarized, broad band, wide beam microstrip patch antenna for navigation terminal applications [7651-22]

T. Dong, China Academy of Space Technology (China); Y. Chen, Beijing Institute of Technology (China)

7651 1X An improved super-exponential algorithm for blind equalization of satellite SIMO communication systems [7651-117]

D. Wu, B. Wang, W. Gao, W. Tan, Space Star Technology Co., Ltd. (China)

7651 1Y A 3D model retrieval approach based on Bayesian networks lightfield descriptor [7651-39] Q. Xiao, Y. Li, Northwestern Polytechnical Univ. (China)

765112 GPS-based attitude determination using adaptive multiple model [7651-50] D. Zhen, S. Knedlik, O. Loffeld, Univ. of Siegen (Germany)

765120 FDSAC-SPICE: fault diagnosis software for analog circuit based on SPICE simulation [7651-162]

Y. Cao, East China Jiaotong Univ. (China); Z. Cen, J. Wei, Huazhong Univ. of Science and Technology (China) 
765121 Design of a general portable communication signal simulator [7651-176]

Q. Zhu, X. Ruan, G. Jin, S. Li, Naval Univ. of Engineering (China)

765122 High speed and bi-mode image compression core for onboard space application [7651-25]

H. Wang, J. Chen, Ctr. for Space Science and Applied Research (China) and Graduate Univ. of the Chinese Academy of Sciences (China); X. Gu, X. Chen, Ctr. for Space Science and Applied Research (China)

765123 A novel high-gain wide-band omnidirectional antenna for satellite communication application [7651-153]

Y. Wang, Space Star Technology Co., Ltd. (China)

765124 Application and research of multi-thread technology in spaceborne SAR imaging [7651-86] L. Tian, S. Fan, W. Zhao, Beijing Institute of Satellite Information Engineering (China)

765125 A new corner detection method based on differential code [7651-116]

S. WU, X. YU, M. XU, Institute of Surveying and Mapping, Information Engineering Univ. (China)

765126 Development and application prospects of modern small satellite technology [7651-70] Y. Wang, Meteorology and Hydrology Bureau of GSD (China); Y. Huang, PLA Univ. of Science and Technology (China)

\section{Part Two}

\section{SATELLITE NAVIGATION AND CONTROL TECHNOLOGY/EARTH OBSERVATION TECHNOLOGY BY} SATELLITES

765127 High-gain integral dynamic compensation robust control for a satellite with mobile body [7651-82]

Z. Zhang, Beijing Institute of Control Engineering (China) and National Lab. of Space Intelligent Control (China); J. Hu, Beijing Institute of Control Engineering (China)

765128 Design of a delay/Doppler mapping receiver for GPS remote sensing [7651-04] W. Li, D. Yang, Beihang Univ. (China); M. Zhang, Space Star Technology Co., Ltd. (China); Q. Zhang, Beihang Univ. (China)

765129 Method of designing GNSS continuously operating reference station network and selecting station place [7651-71]

F. Ke, Q. Wang, S. Pan, Southeast Univ. (China)

7651 2A Partial search for multi-carrier GNSS ambiguity resolution [7651-06]

X. Zhao, Q. Wang, S. Pan, Y. Zhao, Southeast Univ. (China) and European-Asian Educational \& Application Development Ctr. of Galileo (China)

7651 2B Implementation and verification of an advancement to the Galileo system search and rescue service signal simulator [7651-97]

M. Lin, Space Star Technology Co., Ltd. (China); Q. Wu, Beijing Institute of Technology

(China); S. Liu, Space Star Technology Co., Ltd. (China) 
$76512 \mathrm{C}$ Cooperative acquisition algorithms for new GNSS signals [7651-77]

S. Han, Q. Wan, W. Wang, W. Meng, Harbin Institute of Technology (China); Y. Zhang, Space Star Technology Co., Ltd. (China)

7651 2D FFT-based L1 C/A and L2C combined acquisition techniques [7651-28]

S. Han, R. Ma, W. Wang, W. Meng, Harbin Institute of Technology (China); Y. Zhang, Space Star Technology Co., Ltd. (China)

$76512 \mathrm{E}$ Analysis and validation for the compatibility of GPS and Galileo signals [7651-20] S. Zhang, T. Ju, China Academy of Space Technology (China)

$76512 \mathrm{~F} \quad$ Enhancement of satellite VLBI tracking system with capability of correlator model parameters prediction [7651-37]

F. Shu, Y. YU, W. Zheng, X. Zhang, Shanghai Astronomical Observatory (China)

$76512 \mathrm{G}$ Research on the disposition of reference station for SBAS in China [7651-147]

S. Gao, R. Li, Z. Huang, L. Luo, Beihang Univ. (China)

$76512 \mathrm{H} \quad$ Algorithm of multi-path signals restraining of BD receiver based on cepstral [7651-169] J. Wang, W. Liang, J. Yu, J. Yang, National Key Lab. of Science and Technology on C4ISR (China)

$765121 \quad$ An efficient algorithm for autonomous orbit determination of navigation constellation based on cross-link range [7651-44]

H. Li, H. Wang, B. Xu, Nanjing Univ. of Aeronautics and Astronautics (China)

$76512 \mathrm{~J} \quad$ Research on long-term autonomous orbit determination for navigation constellation using inter-satellite orientation observation information [7651-43]

B. Li, B. Xu, H. Wang, Nanjing Univ. of Aeronautics and Astronautics (China)

7651 2K Design method of a baffle of the large-FOV optical system with 2D scan mirror [7651-85] X. L. Li, H. Liu, X. P. Li, Y. T. Pei, Shanghai Institute of Technical Physics (China)

$76512 \mathrm{~L}$ Design of hardware correlator for $\operatorname{BOC}(1,1)$ baseband signal [7651-65]

D. Yang, W. Li, X. Liu, Y. Feng, Beihang Univ. (China)

$76512 \mathrm{M} \quad$ Anti-aliasing filter design on spaceborne digital receiver [7651-87]

D. Yu, C. Zhao, National Key Lab. of Science and Technology on Millimeter Wave System (China)

$76512 \mathrm{~N}$ The research of high accuracy autonomous navigation in the satellite orbit maneuver [7651-104]

L. Wang, Z. Wang, Northwestern Polytechnical Univ. (China)

765120 A new autonomous celestial navigation method based on UKF and MPF [7651-93]

F. Xu, Beijing Aerospace Automatic Control Institute (China)

7651 2P Fixed-point auto-landing algorithm for UAV based on point tracking [7651-108]

Z. Shao, Beijing Institute of Technology (China); Z. Nie, Beihang Univ. (China); Y. Feng,

S. Feng, Beijing Institute of Technology (China) 
$76512 Q$ Relative geometric projection method and argument rotation algorithm for compensation part of an image navigation and registration system [7651-46]

Y. Ning, Z. Wang, W. Li, Northwestern Polytechnical Univ. (China)

7651 2R A low power wide bandwidth CMOS folded-cascode amplifier [7651-115] J. Sun, X. Zhang, Beijing Univ. of Aeronautics and Astronautics (China)

$76512 S$ The evaluation methods of sampling rate performance in GNSS receiver [7651-55]

T. Ke, X. Hu, Y. Liu, Y. Ran, Huazhong Univ. of Science and Technology (China)

$76512 T$ Design and implementation of the correlator used for high dynamic satellite receivers [7651-35]

Q. Zhang, X. Zhang, X. Chang, P. Gong, Beijing Univ. of Aeronautics and Astronautics (China)

$76512 U$ A GPS based autonomous attitude determination and nonlinear adaptive robustness controller design for spacecraft [7651-113]

Y. Fan, Y. Han, Nanjing Univ. of Aeronautics and Astronautics (China); C. Zhou, People's Liberation Army (China)

$76512 \mathrm{~V}$ Vision aided inertial navigation for autonomous pinpoint planetary landing [7651-45]

J. Feng, P. Cui, H. Cui, Harbin Institute of Technology (China)

$76512 \mathrm{~W}$ The mobile LBS positioning solution based on NRTK and Windows Mobile [7651-98] Z. Liao, X. Duan, J. Lu, X. Yue, W. Liu, Q. He, Changsha Univ. of Science and Technology (China)

$76512 X$ The theory and application of the SSDM methodology for solving the deformation information at single epoch by GPS technique [7651-94]

X. YU, W. Lü, Anhui Univ. of Science and Technology (China); S. Xu, Wuhan Univ. (China)

$76512 Y \quad$ A smart broadband satellite system with elastic capacity [7651-69]

S. Feng, Q. Feng, Y. Lin, G. Li, PLA Univ. of Science and Technology (China)

765133 Torque compensation technology for the geostationary meteorological satellite [7651-107] Z. Wang, L. Wang, S. Chen, Northwestern Polytechnical Univ. (China); Q. Li, Shanghai Academy of Spaceflight Technology (China)

\section{SATELLITE SYSTEM AND SYSTEM ENGINEERING TECHNOLOGY}

765134 An efficient algorithm for encoding and decoding of raptor codes over the binary erasure channel [7651-64]

Y.-H. Zhang, B.-W. Cheng, Space Star Technology Co., Ltd. (China) and Peking Univ. (China);

G. Zou, Space Star Technology Co., Ltd. (China); W.-P. Wen, S.-H. Qing, Peking Univ. (China)

765135 Study on method of autonomous orbit determination of navigation satellites based on least-square collocation [7651-112]

W. Liu, X. Gong, Z. Li, F. Wang, Wuhan Univ. (China) 
765136 Application of virtual method in thermal vacuum test of satellite paraboloid antenna [7651-18]

Q. Dou, H. Pang, China Academy of Space Technology (China); Z. Cao, Beijing Institute of Satellite Environment (China); C. Wei, China Academy of Space Technology (China)

765137 Research and embedded implementation of Layer 3 switch [7651-158]

J. Song, Z. Cheng, Space Star Technology Co., Ltd. (China)

765138 Application of infrared lamp array in the thermal balance test of SINOSAT-2 [7651-99] S. Liu, Beijing Univ. of Aeronautics and Astronautics (China); Y. Pei, Beijing Institute of Spacecraft Environment Engineering (China); L. Sun, Beijing Univ. of Aeronautics and Astronautics (China)

765139 The study on integrated mode of inter-satellite communication/measurement in autonomous formation flyer [7651-78]

X. Li, L. Liu, Y. Yang, Univ. of Electronic Science and Technology of China (China)

7651 3A Analysis for scattering of plasma sheath of aircraft by ZT-FDTD method in three-dimensional space [7651-17]

Y. Yang, Z. Wei, M. Fang, G. Chen, Z. Zhang, Nanjing Univ. of Aeronautics and Astronautics (China)

7651 3B Discussion on spacecraft local area networks [7651-175]

W. Zheng, Z. Cheng, J. Liu, Beijing Institute of Satellite Information Engineering (China);

H. Jiang, K. Hu, Beihang Univ. (China)

$76513 \mathrm{C}$ A method for the orbit determination accuracy improvement of a station with a limited motion antenna [7651-154]

F. Wu, Graduate Univ. of the Chinese Academy of Sciences (China), National Time Service Ctr. (China), and Beijing Aerospace Control Ctr. (China); J. Liu, Y. Liu, Y. Li, P. Zhu, L. Du, Beijing Aerospace Control Ctr. (China)

7651 3D Optimization of regional navigation satellite constellation by improved NSGA-Il algorithm [7651-47]

H. Chang, X. Hu, Y. Zhang, Y. Zeng, Y. Wang, Huazhong Univ. of Science and Technology (China)

$76513 \mathrm{E}$ Compensating method for splitter in multi-channel off-axis three-mirror optical system with large field and small $\mathrm{F}$ number [7651-62]

H. Lui, X. Li, Y. Pei, Shanghai Institute of Technical Physics (China)

$76513 \mathrm{~F}$ Simulation of echoes for the design of precipitation radar [7651-88]

W. Xiang, D. Yu, National Key Lab. of Science and Technology on Millimeter Wave System (China); Z. Ge, China Aerospace and Industry Corp. (China)

$76513 G$ Scheme of disaster monitoring competence configured for Chinese space system [7651-165]

Y. Huang, P. Liu, Y. Hou, Y. Fan, Y. Zhou, China Academy of Space Technology (China)

$76513 \mathrm{H} \quad$ A novel on-board switch scheme based on OFDM [7651-83]

J. Dang, P. Zhou, Z. Cao, Tsinghua Univ. (China) 
765131 The application of spread-spectrum system in the area of remote space exploration [7651-156]

F. Wang, X. Wang, China Academy of Space Technology (China) and Space Star Technology Co., Ltd. (China)

7651 3J Development of a FPGA\&DSP-based experimental GNSS receiver platform [7651-151] Y. Hu, Q. Zhang, D. Yang, Beihang Univ. (China)

7651 3K Denoising of ECG signal during spaceflight using singular value decomposition [7651-103] Z. Li, L. Wang, China Academy of Space Technology (China)

$76513 \mathrm{~L} \quad$ Solar activity and space weather changes [7651-101]

A. A. Hady, Cairo Univ. (Egypt)

$76513 \mathrm{M}$ The ring problem of $(\mathrm{N}+1)$ bodies: ten years of research (1999-2009) [7651-138] T. Kalvouridis, National Technical Univ. of Athens (Greece)

$76513 \mathrm{~N}$ Research on remote synchronization system for onboard crystal oscillators of quasi-zenith satellite system [7651-136]

T. Iwata, National Institute of Advanced Industrial Science and Technology (Japan)

765130 Solar power satellites: our next generation of satellites will deliver the sun's energy to Earth [7651-143]

D. M. Flournoy, Ohio Univ. (United States)

$76513 \mathrm{P}$ Space-based solar power generation using a distributed network of satellites and methods for efficient space power transmission [7651-51]

R. M. McLinko, B. V. Sagar, Massachusetts Institute of Technology (United States)

$76513 Q \quad$ Aerospace concurrent engineering: a modern global approach [7651-80]

M. Imbert, X. Li, Beijing Univ. of Aeronautics and Astronautics (China)

7651 3R Design and implementation of spatial situation simulation system based on STK [7651-159] Z. Chen, Wuhan Univ. (China); Y. Meng, J. Mao, Second Artillery Command College (China)

7651 3S A real-time fault diagnosis method of SACS based on combination of offline identification and online observing [7651-119]

Z. Cen, J. Wei, R. Jiang, X. Liu, Huazhong Univ. of Science and Technology (China)

$76513 \mathrm{U}$ A distributed MDO architecture and its application on small satellite [7651-36]

Z. You, G. Li, J. Fu, Tsinghua Univ. (China)

$76513 \mathrm{~V}$ Application and implementation of 1553B protocol and CCSDS protocol in the precipitation radar [7651-90]

Z. Li, D. Yu, National Key Lab. of Science and Technology on Millimeter Wave System (China)

7651 3W Development of a software-based IF GPS signal simulator [7651-106]

Y. Hu, D. Yang, Y. Zhang, Q. Zhang, Beihang Univ. (China)

Author Index 
Downloaded From: https://www.spiedigitallibrary.org/conference-proceedings-of-spie on 26 Apr 2023

Terms of Use: https://www.spiedigitallibrary.org/terms-of-use 


\title{
Conference Committee
}

\author{
Conference Chairs \\ Xingrui Ma, China Aerospace Science and Technology Corporation \\ (China) \\ Don M. Flournoy, Ohio University (United States)
}

Program Committee

Baohua Yang, China Academy of Space Technology (China)

Zhi Li, Second Academy of China Aerospace Science and Industry Corporation (China)

Zhongbao Li, China Academy of Space Technology (China)

Martin C. E. Huber, European Space Agency (France)

Ahmed Abdel Azim Abdel Hady, Cairo University (Egypt)

Leonid Nikolaevich Berdnikov, Moscow University (Russian Federation)

Sabit Ilyasov, Uzbekistan National University (Uzbekistan)

Tilemahos Kalvouridis, National Technical University of Athens (Greece)

Toshiaki Iwata, National Institute of Advanced Industrial Science and Technology (Japan)

Paolo Villoresi, Universitá degli Studi di Padova (Italy)

Jiancheng Shi, University of California, Santa Barbara (United States)

Romina Acevedo, The Bolivarian Agency for Space Activities (Venezuela)

Rustann Dagkesamanskii, P.N. Lebedev Physical Institute, Russian Academy of Sciences (Russian Federation)

Valeriy Orlenko, Kharkov University of Air Forces (Ukraine)

M. B. Rajani, Indian Institute of Science, Bangalore (India)

Wolfgang Kainz, University of Vienna (Austria)

Ohkami Yoshiaki, Japan Aerospace Exploration Agency (Japan)

Ryan M. McLinko, Massachusetts Institute of Technology (United States)

Erik Høg, Danish Astronautical Society (Denmark)

Peijian Ye, China Academy of Space Technology (China)

Shijun Ma, China Academy of Space Technology (China)

Qingjun Zhang, China Academy of Space Technology (China)

Yanguang Wang, China Academy of Space Technology (China)

Liangdong Liu, China Academy of Space Technology (China)

Shan Zhong, Second Academy of China Aerospace Science and Industry Corporation (China)

Ruisong Huang, Third Academy of China Aerospace Science and Industry Corporation (China)

Jianhua Lu, Tsinghua University (China) 
Jingshan Jiang, Chinese Academy of Sciences (China)

Zhongzhao Zhang, Harbin Institute of Technology (China)

Shu Wang, Huazhong University of Science and Technology (China)

Shenglian Luo, Beijing University of Aeronautics and Astronautics

(China)

Wenhe Liao, Nanjing University of Aeronautics and Astronautics (China)

Jun Zhou, Northwestern Polytechnical University (China)

Zongchuang Liang, China Academy of Space Technology (China)

Executive Committee

Ming Li, China Academy of Space Technology (China)

Haitao Wang, Space Star Technology Company, Ltd. (China)

Gang Ao, Second Academy of China Aerospace Science and

Industry

Corporation (China)

Jilin Li, Space Star Technology Company, Ltd. (China)

Haifeng Song, Space Star Technology Company, Ltd. (China)

Chunping Lu, China Academy of Space Technology (China)

Pingmei Hou, Second Academy of China Aerospace Science and Industry Corporation (China)

Jiaolong Wei, Huazhong University of Science and Technology (China)

Conference Secretariat

Bin Shen, China Academy of Space Technology (China)

Weiguang Cui, China Academy of Space Technology (China)

Ning Zhang, China Academy of Space Technology (China)

Dongmei Wu, China Academy of Space Technology (China)

Chunze Zhang, Space Star Technology Company, Ltd. (China)

Nannan Qiu, Space Star Technology Company, Ltd. (China)

Min Yun, Space Star Technology Company, Ltd. (China)

Manjie Zhu, Space Star Technology Company, Ltd. (China)

ShuXia Gao, Second Academy of China Aerospace Science and

Industry Corporation (China)

Session Chairs

$1 \quad$ Satellite System and System Engineering Technology

Don M. Flournoy, Ohio University (United States)

Qingjun Zhang, China Academy of Space Technology (China)

Lihua Zhang, China Academy of Space Technology (China)

2 Satellite Navigation and Control Technology/Satellite Earth

Observation Technology

Ping Shuai, China Academy of Space Technology (China)

Chunling Wei, China Academy of Space Technology (China)

Wolfgang Kainz, University of Vienna (Austria) 
3 Satellite Communication Technology/Satellite Application Technology Yanguang Wang, China Academy of Space Technology (China)

Jilin Li, Space Star Technology Company, Ltd. (China)

Paolo Villoresi, Universitá degli Studi di Padova (Italy)

4 Deep Space Exploration and Communication Technology/Space Information Acquisition, Processing and Application Technology Peijian Ye, China Academy of Space Technology (China)

Zongchuang Liang, China Academy of Space Technology (China)

Martin C. E. Huber, European Space Agency (France)

Jiancheng Shi, University of California, Santa Barbara (United States) 
Downloaded From: https://www.spiedigitallibrary.org/conference-proceedings-of-spie on 26 Apr 2023

Terms of Use: https://www.spiedigitallibrary.org/terms-of-use 


\section{Introduction}

Humankind never gives up their efforts in exploring, developing, and utilizing space. Research and practice have been carried out by generation after generation. Getting rid of the constraints of Earth, humans can observe the Earth from space and understand it from the view of space information. The combination and penetration of modern space technology and information science and technology create new space information science and technology.

With the continuous progress of global space technology and the coming era of space economy, space information technology develops quickly with various new theories and techniques. Space information technology, based on the core technologies of remote sensing, geographical information system (GIS), and global navigation satellite system and satellite communication, is the most important way for information transmission and quick acquisition of the dynamic Earth status of large areas and position information.

Based on the satellite Earth observation, people begin to observe the Earth continuously. Through the information processing which fast reappears and objectively reflects the Earth surface status, phenomena, process, spatial distribution and position. By realizing the global communication with satellite in different ways, space information technology has rendered service to every aspect of national economy construction and social development. Space information technology has become one of the three pioneer scientific areas of the world, in parallel with nanotechnology and biological technology.

Currently, space information technology is quickly applied to the traditional industries. It plays an increasingly important role in the information system reformation in the field of agriculture, urban and regional planning management, transportation, business, and traditional manufacturing, as well as in national macro planning, management, decision-making and social services. The space information high-tech industry has become the pioneer and important part of the information industry. With the rapid development and mutual combination of space technology and its related advanced technologies, space information technology is sure to evolve into a new focus in the future.

Space information technology is highly comprehensive and synthetic, which requires the close collaboration among the institutions of payload development, satellite manufacture and launch, satellite data receiving and processing, and application. The China Aerospace Science and Technology Corporation, as the backbone of China's space technology research, is dedicated to launch vehicles, satellite and spin-off, all of which have close relationship with space information technology. This technology covers all the phases of space information acquisition, transmission, processing and application. In order to 
enhance satellite application, satellite ground operation service, information technology and other business, the Satellite Application Academy has been established this year, whose aim is to utilize the research outcomes of space technology for sustainable development of national economy and the society, and to promote the research and application of space information technology in China.

The China Aerospace Science and Technology Corporation will always welcome all the experts and friends. We expect your visit to CASC for information exchange and cooperation. I believe all our friends at this conference will make joint efforts with us to contribute to the development of space information technology.

Xingrui Ma 